\title{
Análise das interaçõesdos alunos com Conteúdos e Atividades em Ambientes Colaborativos Virtuais de Aprendizagem
}

\author{
Jarbele C. Silva ${ }^{1}$, Alisson V. Brito ${ }^{1}$, Fellipe Mahon ${ }^{1}$, Francisco P. A. de Medeiros ${ }^{2}$ \\ ${ }^{1}$ Centrode Informática - Universidade Federal da Paraíba (UFPB) \\ João Pessoa - PB - Brasil \\ ${ }^{2}$ Instituto Federal de Educação, Ciência e Tecnologia da Paraíba (IFPB) \\ João Pessoa, PB - Brasil \\ jarbele.cassia@gmail.com, alisson@ci.ufpb.br, fellipe.mahon@gmail.com, \\ petroniodifpb.edu.br
}

\begin{abstract}
This article presents the results of studies on the interaction between students and access to the contents of a Bachelor's Degree in Computer Science in the distance of XXXX University. Its purpose is, through the metric of Social Network Analysis, ascertain whether this type of online interaction can impact, positively or not, the student's performance in courses of this type.
\end{abstract}

Resumo. Este artigo apresenta o relato dos resultados deestudos realizados acerca da interação existente entre alunos e o acesso ao conteúdo de um curso de Licenciatura emCiência da Computaçãonamodalidade a distância da Universidade XXXX.Seu propósito consiste em, por meiodasmétricas de Análise de Redes Sociais, averiguar se este tipo de interação online pode impactar, positivamente ou não, no desempenho do aluno em cursos desta modalidade.

\section{Introdução}

A utilização das redes sociais mediadas pela Internet além de proporcionarem um ambiente propício ao compartilhamento de informações atualmente tem assumido um caráter interativo.Diante desta nova percepção foi notado que o agrupamento em redes sociais online também é construído no âmbito educativo, sobretudo em modelos de ensino à distância (E-Learning). Neste formato de ensino, as pessoas não estão apenas reunidas em torno de um tema. Há uma proposta a cumprir, situações didáticas e alguém do grupo que provoca e estimula a participação dos demais.

Visando, portanto, complementar as pesquisas que relacionamAnálise de Redes Sociais (SNA) e E-learning, este trabalho édirecionadoàinvestigação, análise e discussão acercadas possibilidades de estudo sobre como uma abordagem de SNA pode contribuir noexame da interação entre alunos e o acesso ao conteúdo em Ambientes Colaborativos Virtuais de Aprendizagem (ACVA), de modo a apontarindícios de bom desempenho, ou não, dos alunos de um curso de Licenciatura Ciência da Computaçãona modalidade à distância.

A seção 2 apresenta os trabalhos relacionados a esta temática. A seção 3 contextualiza brevemente aAnálise de Redes Sociais. A seção 4 expõe a metodologia utilizada para obtenção dos dados. A seção 5 apresenta a descrição do estudo de caso realizado. E, por fim, a última seção aborda as consideraçõesfinais e a proposta de trabalhos futuros. 


\section{Trabalhos Relacionados}

Em seu trabalho, Silva e Brito (2014) apresentamos resultados de um Levantamento Bibliográfico, na língua inglesa, acerca dos artigos científicos que versam sobre a SNA aplicada em modelos de ensino E-Learning. Foi percebido, portanto, que existem caminhos ainda pouco explorados no que se refere ao estudo da interação entre alunos e o acessoaoconteúdo de um curso à distância, segundo as métricas de Análise de Redes Sociais.Segundo este trabalho, pesquisassobre a temática em foco tornam-se viáveis, visto as possibilidades de estudo existentes para tal.

Vieira (et. al., 2012) destaca a importância na compreensão das interações no seio de uma comunidade de aprendizagem e define três tipos de interação: aprendente/conteúdo; aprendente/tutor; aprendente/aprendente. Ele enfatiza que a interação aprendente/conteúdo constitui um elemento-chave da aprendizagem, uma vez que o processo de interagir intelectualmente com o conteúdo resulta numa mudança na estrutura cognitiva do aprendente.

Em seu estudoMansur \& Yusof(2013)apresentam um estudo relacionado à SNA aplicada em E-learning buscando evidenciar o comportamento de alunos nas atividades de um sistema E-Learning (Moodle) através da análise das ações desempenhadas pelos estudantes em cada atividade. Por fim, baseado em técnicas de Clustering, ele define o comportamento dos estudantes, classificando-os em: ativos, construtivos, intencionais, autênticos ou cooperativos.

\section{Análise de Redes Sociais}

Através da Análise de Redes Sociais é possívelcompreender e acompanhar de forma mais eficaz a disseminação de informações e a interação entre aspessoas que compõem a rede [Oliveira, et. al. 2012]. Para identificar e interpretar os relacionamentos existentes em redes sociais online faz-se necessário o uso de métricas de SNA específicas.

No âmbito da interação entre aluno e conteúdo, foco desse estudo, os dados são melhorrepresentados em uma matriz de adjacência (aqui chamada Matriz de Interação), visto que a matriz atende com mais precisão os questionamentos da pesquisa, refletindo de modo mais objetivo e claro a frequência das ocorrências de acesso aos conteúdos/atividade pelos alunos do curso à distância. Nesse caso, o peso da relação, ou seja interação, é dado pelo valor $[\mathrm{i}, \mathrm{j}]$, onde: $[\mathrm{i}, \mathrm{j}]>0$, informa que existe ligação de i a j; quando [i,j] é 0 implica dizer que não existe ligação de i a j. Desta forma, quanto mais valor entre i e j, maior o peso da relação entre os nós.

\section{Metodologia}

O estudo proposto para SNA com basenas interações do Moodleé apoiado em um conjunto de atividades que objetivam mapear o desempenho dos alunos de um curso de Licenciatura em Ciência da Computação à distância através das interações estabelecidas entre os estudantes e o acesso ao conteúdo. Deste modo, são apresentados a seguir os procedimentosutilizados na pesquisa.

$\mathrm{Na}$ fase de Planejamento são traçados os objetivos do estudo e definidos os atributos que serão observados no Monitoramento, a citar: os grupos de alunos a serem avaliados; o tipo de conteúdo a analisar; a disciplina do curso; e, o semestre.A escolha do Polo e disciplina obedeceram a alguns requisitos experimentais: maior quantidade de estudantes matriculados na disciplina; disponibilidade do resultado dos 
exercíciosavaliativos feitos pelos alunos; bem como, o maior número de recursos do ACVA utilizados. Com base nesses critérios, escolheu-se o polo pertencente à região $\mathrm{X} \mathrm{e}$ a disciplina de Introdução à Programação. Foram selecionados, também, os conteúdos Vídeo e Apostila e a atividade Exercício de Envio, ambos referentes à mesma temática de estudo ou Unidade da disciplina.

A fase de Monitoramento é destinada à coleta de dados que servirá de base para a realização desse estudo de caso. Deste modo, os atributos definidos na fase de Planejamento são extraídos do relatório de logs da plataforma Moodle, no formato de um arquivo XML. Foram consideradosos dados referentes ao nome do aluno (representados por: 1 (aluno 1), 2 (aluno 2), consecutivamente) a ação e a informação registradas. Por motivos de privacidade os nomes dos alunos foram omitidos.

A fase de Modelagem de interação é iniciada a partir do arquivo gerado na fase anterior. É, então, elaborada uma matriz de interação, com os dados referentes aos conteúdos (Vídeo e Apostila) e a atividade(Exercício de Envio) que indica e representa as interações existentes entre os alunos e o tipo de conteúdo monitorado.Cada interação foi quantificada por meio da representação na matriz, onde $i$ representa os alunos e $j$ o conteúdo ou atividade em análise. Quando a $[\mathrm{i}, \mathrm{j}]>0$, temos uma interação do usuário $i$ para o conteúdo/atividade $j$, indicando também a quantidade de acessos deste estudante ao conteúdo/atividade. Em contrapartida, quando a $[i, j]=0$ inferimos que não existe interação entre os mesmos.

$\mathrm{Na}$ fase de Análise os resultados obtidos são tratados minuciosamente com o intuito de perceber a influência do acesso a um dado conteúdo sobre o desempenho dos alunos. Esta fase é descrita na seção a seguir.

\section{Estudo de caso}

Sabendo que a atividade valia de 0 a 2,0 pontos foi atribuído a ela alguns conceitos de acordo com a pontuação obtida por cada aluno, a listar: ruim $(0-0,5$ pontos $)$, regular $(0,5-1,0$ pontos $)$, bom $(1,0-1,5$ pontos $)$, ótimo (1,5 - 1,7 pontos) e excelente $(1,8$ 2,0pontos). Foi, então, identificado o desempenho dos alunos no "Exercício de Envio" e constatado que cerca de $53 \%$ dos aluno (equivalente a 18) alcançaram um desempenho excelente, $9 \%$ deles(equivalente a 3) obtiveram um ótimo desempenho na atividade, enquanto que 3\% (1 aluno) alcançou um bom desempenho eos outros 35\% (12 alunos) apresentaram um desempenho ruim. É importante mencionar que nenhum deles obteve um desempenho regular na atividade.

Em seguida, o resultado obtido pelos alunos na atividade foi comparado com as suas respectivas interações com os conteúdos Vídeo e Apostila, representados na matriz de interação. Desta maneira percebeu-se que os alunos que interagiram (acessaram ou realizaram o download) com mais frequência com os conteúdos supracitados, e referentes ao tema proposto à semana de estudo, obtiveram um desempenho melhor que os demais. A Tabela 1 apresenta esta informação. 
Tabela 1. Comparativo entre a interação dos alunos com os conteúdos "Vídeo" e "Apostila" e o desempenho na atividade "Exercício de Envio"

\begin{tabular}{|c|c|c|}
\hline Interação (Vídeo+Apostila) & Quantidadede alunos & $\begin{array}{c}\text { Média doDesempenho } \\
(\mathbf{0}-\mathbf{2 , 0})\end{array}$ \\
\hline Apenas 1 vez & 3 & 0,66 \\
\hline Apenas 2 vezes & 8 & 0,92 \\
\hline De 3 a 4 vezes & 13 & 1,09 \\
\hline 5 a maisvezes & 10 & 1,37 \\
\hline
\end{tabular}

É válido ressaltar que dentre os dez alunos (citados na Tabela 1) que interagiram 5 ou mais vezes, oito deles obtiveram um desempenho excelente, um deles alcançou um desempenho ótimo e, um último interagiu essa quantidade de vezes, mas não chegou a realizar o exercício de envio.

Percebeu-se ainda, que os alunos 4, 2 e 20 (aproximadamente 9\% dos alunos) na matriz de interação possuem os maiores índices de acesso, e coincidentemente, são alguns dos alunos que obtiveram as maiores pontuações no exercício avaliativo. Deste modo podemos afirmar, para este caso, que aqueles alunos que interagem com os mais variados conteúdos tem uma maior probabilidade de alcançar um melhor desempenho nas atividades da disciplina.

Por outro lado, percebeu-se queos menores índices de acesso foram dos alunos5, 9 e 27 (cerca de 9\% dos alunos), sendo que, apenas um deles conseguiu alcançar um excelente desempenho na atividade. É importante mencionar que oaluno5 surpreendeu positivamente, visto que obteve um excelente desempenho no exercício, mesmo interagindo poucas vezes com o conteúdo. Esse fato se deve a prática constante de exercícios anteriores, identificados no relatório de registros do Moodle, feitos pelo aluno em destaque.

Notou-se também que os alunos3, 6, 7, 9, 11, 17, 23, 27, 28, 30, 31 e 32 (equivalente a $35 \%$ dos alunos) da matriz de interação, não realizaram o envio do exercício avaliativo, apenas acessaram o conteúdo. Não foram registrados casos em que o aluno tenha realizado o envio do exercício sem acessar o conteúdo em análise.

Esses fatores nos permitem presumir que, se um aluno interage menos com os conteúdos disponíveis no ACVA, a probabilidade de não conseguir realizar as atividades avaliativas ou alcançar bons resultados nelas é maior. Portanto, através da matriz de interação é possível identificar, neste contexto, os possíveis fatores que influenciam positivamente, ou não, no desempenho de alunos de um curso à distância.

\section{Considerações Finais}

Neste trabalho foi apresentada a análise da interação entre alunos e o acesso ao conteúdo no Ambiente Colaborativo Virtual de Aprendizagem Moodle, em específico, por meio de um estudo de caso realizado em um curso de Licenciatura em Ciência da Computação à distância. Os resultados preliminares apontam que,por meio de métricas de SNA é possível presumir se as interações estabelecidas com o conteúdo de uma determinada disciplina poderão contribuir e/ou impactar no desempenho das atividades realizadas pelos alunos ao longo do curso da mesma.

Nesse contexto é proposta para trabalhos futuros a realização de experimentos com um número maior de alunos e conteúdos, fazendo uso das métricas de SNA cabíveis a análise das interações existentes neste meio, com o intuito de identificar a 
existência de relações diretas ou indiretas de um aluno com outro através do conteúdo e/ou atividade acessado por ambos. Desta forma, seria possível entender a existência de interações sociais mediadas por um determinado conteúdo.

Também é proposta possibilidades de definir, ou inferir, o perfil dos alunos e/ou aqueles que tendem a evadir de um curso à distância, por meio da análise das interações com o conteúdo, buscando justificar a influência do conteúdo neste tipo de ocorrência.

\section{Referências}

Campana, V. F., Sanches, D. R. e Tavares, O. L., Souza, S. F. (2008) "Agentes para Apoiar o Acompanhamento das Atividades em Ambientes Virtuais de Aprendizagem", Simpósio Brasileiro de Informática na Educação (SBIE), Fortaleza.

Magalhães, A., Oliveira, G.,Fialho, S. (2012)“Indicadores em educação a distância: o uso da matriz de necessidades para ambientes virtuais de aprendizagem", XVII Congresso Internacional de Informática Educativa, TISE, Santiago, Chile pp. 409-412.

Mansur, A. B. F., Yusof, N. (2013)“'Social learning network analysis model to identify learning patterns using ontology clustering techniques and meaningful learning", Computers\& Education.

Medeiros, F., Gomes, A. S., Amorim, R., Medeiros G.(2013) "Architecture for Social interactions Monitoring in Collaborative Learning Enviroments as a Supportfor the Teacher's Awareness", In Advanced Learning Technologies (ICALT), IEEE 13th International Conference on (pp. 123-127).

Medeiros, F. P. A., Gomes, A. S. (2012) "Monitoramento da Experiência do Usuário em Ambientes Colaborativos Virtuais de Aprendizagem: um Mapeamento Sistemático", In: Anais do Simpósio Brasileiro de Informática na Educação (vol. 23, No.1)

Oliveira, R., Araújo, J.,Medeiros, F., Brito, A. (2012)“Monitoramento das Interações dos Aprendizes na Rede Social Twitter como Apoio ao Processo de Mediação Docente",Brazilian Workshop on Social Network Analysisand Mining, BrasNAM, XXXII Congresso da Sociedade Brasileira de Computação, CSBC, Curitiba, PR.

Silva, J. C., Brito, A. V. (2014) "Levantamento bibliográfico das publicações acadêmico-científicas sobre Análise de Redes Sociais aplicadas em E-Learning", XXIII Ciclo de Palestras sobre Novas Tecnologias na Educação, CPNTE, Porto Alegre, RS.

Vieira,M. C., Silva, J. C., Mendes, A. J.,Marcelino,M. J., Pessoa, M. T. (2012)“Análise de interações estabelecidas num fórum de aprendizagem do curso de violência e gestão de conflitos na escola”, II Congresso Internacional TIC e Educação. 